\title{
House dust mite SLIT-tablet is well tolerated in pediatric patients with controlled asthma
}

\author{
Hisashi Tanida, ${ }^{1}$ Takayasu Nomura, ${ }^{1}$ Yuto Kondo, ${ }^{1}$ Yasutaka Hirabayashi, ${ }^{2}$ Jun Wakatsuki, ${ }^{3}$ Shinji Saitoh ${ }^{1}$
}

\begin{abstract}
Background: Despite the reported clinical effectiveness of house dust mite (HDM) sublingual immunotherapy (SLIT) in pediatric patients, the risk of treatment remains unclear in pediatric patients with allergic asthma.
\end{abstract}

Objective: To show a risk of adverse drug reactions (ADRs) in pediatric patient with allergic asthma during the initiation period of HDM SLIT.

Methods: We retrospectively analyzed the clinical data of pediatric patients aged $\leq 15$ years who initiated allergen immunotherapy (AIT) with the SQ HDM SLIT-tablet for allergic rhinitis between February 2017 and September 2019. Asthma severity at baseline and ADRs during the first 4 weeks of the treatment were determined for each subject.

Results: In our study population ( $\mathrm{n}=217$; median age, 8.4 years), 99 patients $(45.6 \%)$ were classified as having asthma. One hundred and one patients (46.5\%) in the whole cohort experienced ADRs during the first 4 weeks of therapy, but a major gap in the frequency of ADRs was not observed between an asthma group and a non-asthma group.

Conclusion: The SQ HDM SLIT-tablet was well tolerated in pediatric patients with controlled HDM-driven allergic asthma. HDM-SLIT is an option to treat their allergic rhinitis without excessive concern for its ADRs.

Key words: Adverse reaction, Allergic asthma, Children, House dust mite, Sublingual immunotherapy

\footnotetext{
From:

${ }^{1}$ Department of Pediatrics and Neonatology, Graduate School of Medical Sciences, Nagoya City University, Nagoya, Japan

${ }^{2}$ Hibarigaoka Kids Allergy Clinic, Nagoya, Japan

${ }^{3}$ Wakatsuki Kids Allergy Clinic, Nagoya, Japan
}

\section{Corresponding author:}

Takayasu Nomura

Department of Pediatrics and Neonatology, Graduate School of

Medical Sciences, Nagoya City University

1 Kawasumi, Mizuho, Nagoya, Aichi 467-8601, Japan

E-mail: tnomura@med.nagoya-cu.ac.jp

\begin{tabular}{|c|c|}
\hline \multicolumn{2}{|c|}{ Abbreviations } \\
\hline ADR & adverse drug reaction \\
\hline AIT & allergen immunotherapy \\
\hline HDM & house dust mite \\
\hline SLIT & sublingual immunotherapy \\
\hline
\end{tabular}

\section{Introduction}

House dust mite (HDM) is a major cause of allergic rhinitis. The standardized quality (SQ) HDM sublingual immunotherapy (SLIT)-tablet (TO-203, Torii, Japan/ALK Denmark) was developed as a disease modifier of HDM-driven allergic rhinitis. ${ }^{1}$ Uncontrolled asthma is a contraindication for HDM SLIT because it is a major independent risk factor for severe adverse reactions. ${ }^{2}$ In contrast, because half of the population of patients with allergic rhinitis experience allergic asthma, ${ }^{3}$ the risk of immunotherapy with controlled asthma needs to be determined. A recent subgroup analysis revealed that the treatment was well tolerated in adult patients. ${ }^{3}$ However, the risk of treatment remains unclear in pediatric patients with allergic asthma, despite the reported clinical effectiveness of immunotherapy. ${ }^{4}$ Here, we show that the SQ HDM SLIT-tablet is well tolerated in pediatric patients with controlled allergic asthma.

\section{Methods}

We retrospectively analyzed the clinical data of pediatric patients aged $\leq 15$ years who were administered allergen immunotherapy (AIT) with the SQ HDM SLIT-tablet for allergic rhinitis at 2 pediatric allergy clinics located in Japan (February 2017-September 2019). The SQ HDM SLIT-tablet was indicated for patients with HDM-driven allergic rhinitis with a clinical history and sensitization to the HDM allergen. The SLIT protocol includes daily treatment at a dose of 3,300 JAU (2 SQ-HDM) for one week, and subsequent doses of 10,000 JAU (6 SQ-HDM). ${ }^{1,4}$ At the beginning of the SLIT, 
the asthma status was assessed according to the Japanese guidelines for childhood asthma. " "True" asthma severity was determined based on the frequency and severity of asthma symptoms considering the treatment step and was then classified as intermittent, mild-persistent, moderate-persistent, and severe-persistent asthma. In the present study, patients with intermittent asthma were included in the non-asthma group because these patients are not classified as asthmatic as per other internationally recognized guidelines. Asthma control levels were also classified according to the conditions during the 4 preceding weeks: well-controlled, partially controlled, and poorly controlled. ${ }^{5}$ Because new adverse drug reactions (ADRs) were rarely observed after 5 weeks of treatment initiation, ${ }^{3}$ we focused on the first 4 weeks of the treatment. Therefore, 217 patients were included in this study, after excluding one patient with poorly controlled asthma status at the therapy initiation. This study was approved by a local research ethics committee.

Investigators carefully assessed the severity of all ADRs and their possible relationship with treatment. ADRs were graded according to the grading systems for systemic reactions $^{6}$ and local reactions during SLIT. ${ }^{7}$ The Mann-Whitney and Fisher's exact tests were used to assess differences between the groups. All statistical analyses were performed using PRISM 8 (GraphPad Software, San Diego, California, USA).

\section{Results}

In our study population $(\mathrm{n}=217$; median age, 8.4 years), 99 patients (45.6\%) were classified as having asthma (Table 1A). A lower age limit for the treatment is recommended as 5 years of age in Japan, but the population included three 4 -year-old children. Although the asthma group had more male patients than did the non-asthma group $(67.7 \%$ vs. $53.4 \%, P=0.037)$, no differences were detected in total $\mathrm{IgE}$ $(P=0.86)$ and HDM-specific $\operatorname{IgE}(P=0.77)$. The level of regular use of antihistamines was also similar at the beginning of the SLIT $(P=0.45)$. Then we compared ADRs and the establishment of the regular dose of SLIT-tablet at 4 weeks: 101 patients (46.5\%) in the whole cohort experienced ADRs during the first 4 weeks of therapy (Table 1B). In general, most ADRs were mild, and a major gap in the frequency of ADRs was not observed between the groups. ADRs classified as asthma included 4 patients (4.0\%) from the asthma group and 2 patients (1.7\%) from the non-asthma group. Because we classified self-reported temporal dyspnea as asthma, only 2 patients from the asthma group were clinician-diagnosed mild asthma exacerbations. Furthermore, we confirmed that these 2 patients reached the regular dose of SLIT-tablet without symptoms at 4 weeks of therapy. Regarding to the treatment of ADRs mostly occurred within an hour, no extra visits were needed for inhaled bronchodilator or adrenaline injection.

Table 1A. Subject characteristics

\begin{tabular}{|c|c|c|c|}
\hline & $\begin{array}{l}\text { Asthma } \\
(\mathbf{n}=99)\end{array}$ & $\begin{array}{l}\text { Non-asthma } \\
(\mathrm{n}=118)\end{array}$ & $P$-value \\
\hline Sex, male (\%) & $67(67.7)$ & $63(53.4)$ & $0.037^{*}$ \\
\hline Age, median (quartile), years & $8.4(6.6,10.3)$ & $8.5(6.7,10.8)$ & 0.69 \\
\hline Inhaled corticosteroid (\%) & $51(51.5)$ & $0(0)$ & \\
\hline \multicolumn{4}{|l|}{ True asthma severity } \\
\hline mild-persistent (\%) & $63(63.6)$ & & \\
\hline moderate-persistent (\%) & $29(29.3)$ & & \\
\hline severe-persistent (\%) & $7(7.1)$ & & \\
\hline \multicolumn{4}{|l|}{ Asthma control level } \\
\hline well controlled (\%) & $92(92.9)$ & & \\
\hline partially controlled (\%) & $7(7.1)$ & & \\
\hline $\begin{array}{l}\text { HDM-specific IgE, median } \\
\text { (quartile), IU/mL }\end{array}$ & $\begin{array}{c}64.5 \\
(27.8,100)^{\dagger}\end{array}$ & $\begin{array}{c}57.9 \\
(25.6,100)\end{array}$ & 0.77 \\
\hline $\begin{array}{l}\text { Total IgE, median (quartile), } \\
\text { IU } / \mathrm{mL}\end{array}$ & $\begin{array}{c}488.0 \\
(271.5,988.0)^{\ddagger}\end{array}$ & $\begin{array}{c}556.5 \\
(226.5,1015)^{\varsigma}\end{array}$ & 0.86 \\
\hline Sensitization to pollen (s) & $75(75.7)^{\dagger}$ & $91(77.1)$ & 0.87 \\
\hline $\begin{array}{l}\text { Oral antihistamine at the } \\
\text { beginning of immunotherapy }\end{array}$ & $69(69.7)$ & $88(74.6)$ & 0.45 \\
\hline
\end{tabular}

${ }^{\star} P<0.05$

${ }^{\dagger} 1$ missing datum, "five missing data, ${ }^{\S}$ four missing data;

Abbreviation: HDM, house dust mite 
Table 1B. Adverse drug reactions and dosing of house dust mite sublingual immunotherapy

\begin{tabular}{|c|c|c|c|c|}
\hline & $\begin{array}{c}\text { Overall } \\
(n=217)\end{array}$ & $\begin{array}{l}\text { Asthma } \\
(\mathrm{n}=99)\end{array}$ & $\begin{array}{l}\text { Non-asthma } \\
(\mathrm{n}=118)\end{array}$ & $P$-value \\
\hline \multicolumn{5}{|c|}{ SYSTEMIC REACTIONS, $\%$ ( $\geq$ grade 2 ) } \\
\hline \multicolumn{5}{|l|}{ Conjunctival } \\
\hline - pruritus & $3.2(0)$ & $3.0(0)$ & $3.4(0)$ & $>0.99$ \\
\hline - erythema & $0.9(0)$ & $1.0(0)$ & $0.8(0)$ & $>0.99$ \\
\hline \multicolumn{5}{|l|}{ Cutaneous } \\
\hline - generalized pruritus & $3.2(0)$ & $5.1(0)$ & $1.7(0)$ & 0.25 \\
\hline - urticaria & $0.9(0)$ & $2.0(0)$ & $0(0)$ & 0.21 \\
\hline \multicolumn{5}{|l|}{ Upper respiratory } \\
\hline - cough & $0.9(0)$ & $1.0(0)$ & $0.8(0)$ & $>0.99$ \\
\hline - rhinitis & $5.5(0)$ & $4.0(0)$ & $6.8(0)$ & 0.55 \\
\hline \multicolumn{5}{|l|}{ Lower respiratory } \\
\hline - asthma & $2.8(2.8)$ & $4.0(4.0)$ & $1.7(1.7)$ & 0.42 \\
\hline \multicolumn{5}{|c|}{ LOCAL REACTIONS, \% ( $\geq$ grade 2 ) } \\
\hline \multicolumn{5}{|l|}{ Mouth/ear } \\
\hline - itching of lips & $0.5(0)$ & $0(0)$ & $0.8(0)$ & $>0.99$ \\
\hline - swelling of lips & $0.9(0.5)$ & $1.0(0)$ & $0.8(0.8)$ & $>0.99$ \\
\hline - itching of oral mucosa & $14.7(1.4)$ & $13.1(1.0)$ & $16.1(1.7)$ & 0.57 \\
\hline - swelling of oral mucosa & $5.5(2.3)$ & $3.0(1.0)$ & $7.6(3.4)$ & 0.23 \\
\hline - itching of ears & $14.7(1.4)$ & $14.1(2.0)$ & $15.3(0.8)$ & 0.85 \\
\hline - swelling of tongue & $1.4(0)$ & $1.0(0)$ & $1.7(0)$ & $>0.99$ \\
\hline - throat irritation & $20.7(6.5)$ & $15.2(6.1)$ & $25.4(6.8)$ & 0.07 \\
\hline \multicolumn{5}{|l|}{ Upper gastrointestinal } \\
\hline - nausea & $1.4(0)$ & $2.0(0)$ & $0.8(0)$ & 0.59 \\
\hline \multicolumn{5}{|l|}{ Lower gastrointestinal } \\
\hline - abdominal pain & $0.5(0)$ & $1.0(0)$ & $0(0)$ & 0.46 \\
\hline \multicolumn{5}{|l|}{ REDUCED SLIT DOSING, \% } \\
\hline \multicolumn{5}{|l|}{ (discontinued) } \\
\hline$-<10,000 \mathrm{JAU}$ at 4 weeks & $6.5(0.9)$ & $7.1(0)$ & $5.9(1.7)$ & 0.79 \\
\hline
\end{tabular}

Abbreviations: JAU, Japanese allergy unit; SLIT, Sublingual immunotherapy

Three patients in the asthma group and 8 patients from the non-asthma group were treated with additional oral antihistamines. In contrast, regarding the achievement of the regular dose of SLIT-tablet, 7 patients in both groups $(7.1 \%$ in the asthma group and $5.9 \%$ in the non-asthma group) failed to achieve 10,000 JAU at 4 weeks of therapy and were administered reduced doses (such as 3,300 JAU) except for 2 cases $(1.7 \%)$ who discontinued the therapy in the non-asthma group. The reasons for discontinuation were mild ADRs of upper respiratory symptom and swelling of oral mucosa. It is noteworthy that 3 of 7 patients classified as severe-persistent asthma only experienced mild ADRs with a regular protocol of the therapy.

\section{Discussion}

Our results showed that controlled allergic asthma is not a risk factor for the frequency and severity of ADRs and the achievement of the regular dose of the SQ HDM SLIT-tablet. SQ HDM SLIT-tablet was well tolerated in adult patients with HDM-derived allergic asthma, as assessed by analyzing a subgroup of clinical trials. ${ }^{3}$ Although the retrospective design is a limiting factor, our findings indicate that the SQ HDM SLIT-tablet for allergic rhinitis can be applied to pediatric patients with controlled allergic asthma without excessive concern regarding severe ADRs. 
Concomitantly, successful treatment of HDM-driven allergic asthma is expected after SLIT because HDM is the most important indoor allergen associated with allergic asthma. However, the effectiveness of the SQ HDM SLIT-tablet for allergic asthma is rather complex. ${ }^{8}$ To date, AIT using the HDM SLIT-tablet has yielded a robust effect in adult patients with asthma, whereas the data available for children are insufficient. ${ }^{2}$ Because most data stemmed from retrospective subgroup analyses from AIT trials for allergic rhinitis, from which patients with concomitant asthma were analyzed, further well-designed clinical trials are needed for successfully treating HDM-driven allergic asthma.

\section{Conclusion}

The SQ HDM SLIT-tablet was well tolerated in pediatric patients with controlled HDM-driven allergic asthma. HDMSLIT is an option to treat their allergic rhinitis without excessive concern for its ADRs.

\section{Conflict of interest}

The authors declare that there is no conflict of interest regarding the publication of this article.

\section{Financial support}

None

\section{Statement of Ethics}

This study was approved by a local research ethics committee (Nagoya City University Hospital, \#60-19-0134).

\section{Clinical trial registration}

Not applicable

\section{References}

1. Okubo K, Masuyama K, Imai T, Okamiya K, Stage BS, Seitzberg D, et al. Efficacy and safety of the SQ house dust mite sublingual immunotherapy tablet in Japanese adults and adolescents with house dust mite-induced allergic rhinitis. J Allergy Clin Immunol. 2017;139:1840-8

2. Agache I, Lau S, Akdis CA, Smolinska S, Bonini M, Cavkaytar O, et al EAACI Guidelines on Allergen Immunotherapy: House dust mite-driven allergic asthma. Allergy. 2019;74:855-73

3. Emminger W, Hernández MD, Cardona V, Smeenk F, Fogh BS, Calderon MA, et al. The SQ house dust mite SLIT-tablet is well tolerated in patients with house dust mite respiratory allergic disease. Int Arch Allergy Immunol. 2017;174:35-44

4. Masuyama K, Okamoto Y, Okamiya K, Azuma R, Fujinami T, Riis B, et al. Efficacy and safety of SQ house dust mite sublingual immunotherapy-tablet in Japanese children. Allergy. 2018;73:2352-63

5. Arakawa H, Hamasaki Y, Kohno Y, Ebisawa M, Kondo N, Nishima S, et al. Japanese guidelines for childhood asthma 2017. Allergol Int. 2017;66: 190-204

6. Cox L, Larenas-Linnemann D, Lockey RF, Passalacqua G. Speaking the same language: The World Allergy Organization Subcutaneous Immunotherapy Systemic Reaction Grading System. J Allergy Clin Immunol. 2010;125:569-74

7. Passalacqua G, Baena-Cagnani CE, Bousquet J, Canonica GW, Casale TB, Cox L, et al. Grading local side effects of sublingual immunotherapy for respiratory allergy: speaking the same language. J Allergy Clin Immunol. 2013;132:93-8

8. Calderon MA, Bousquet J, Canonica GW, Cardell L-O, Fernandez de Rojas DH, Kleine-Tebbe J, et al. Guideline recommendations on the use of allergen immunotherapy in house dust mite allergy: Time for a change? J Allergy Clin Immunol. 2017;140:41-52 